\title{
Potential fish habitat mapping using MODIS-derived sea surface salinity, temperature and chlorophyll-a data: South China Sea Coastal areas, Malaysia
}

\begin{abstract}
This paper demonstrates the use of moderate resolution imaging spectro-radiometer (MODIS) data for fish forecasting mapping of seasonal spatial distribution of sea surface salinity (SSS), temperature (SST) and chlorophyll-a in the ocean waters off the coast of Semporna, Malaysia. Multi-linear regression analysis was performed to estimate SSS and the Brown and Minnet algorithm was used for the SST. The extracted parameters were validated using in situ measurement taken with Hydro-Lab equipment. The extracted parameters from MODIS images reveal the signature values which establish the relationships between these parameters, and thus delineating the potential fish zonation (PFZ) map. These developed models will help for accurate monitoring of large coverage areas at low cost and within short period of time. Furthermore, such models will allow the prediction of the total fish catch in different seasons, thus contributing to fish industry management and marketing. This research recommends the use of PFZ map for mass scale fish harvesting in short time for larger areas. Finally, the research has developed a potential fish zone model amalgamating all the above parameters. The PFZ mapping was carried out off the coast of Semporna, Sabah as there were sufficient fish catch data for accuracy assessment. The $\mathrm{R}$ was computed as 0.93 and the higher fish catch areas have coincided very well with the higher PFZ values, meaning the tool is ready for use for operational near real-time fish forecasting.
\end{abstract}

Keyword: Fish forecasting; Remote sensing; Temperature; Salinity; Chlorophylls; Geographic information systems (GIS) 